\title{
Determinants of clinical practice guidelines' utilization for the management of musculoskeletal disorders: a scoping review
}

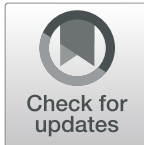

Delphine Sorondo ${ }^{1,2^{*}}$, Cyrille Delpierre ${ }^{1}$, Pierre Côté ${ }^{3,4}$, Louis-Rachid Salmi ${ }^{5,6}$, Christine Cedraschi ${ }^{7,8}$, Anne Taylor-Vaisey ${ }^{3,4}$ and Nadège Lemeunier ${ }^{1,3}$

\begin{abstract}
Context: Many clinical practice guidelines have been developed for the management of musculoskeletal disorders (MSDs). However, there is a gap between evidence-based knowledge and clinical practice, and reasons are poorly understood. Understanding why healthcare providers use clinical practice guidelines is essential to improve their implementation, dissemination, and adherence.

Aim: To identify determinants of clinical practice guidelines' utilization by health care providers involved in the assessment and management of MSDs.

Method: A scoping review of the literature was conducted. Three databases were searched from inception to March 2021. Article identification, study design, methodological quality, type of healthcare providers, MSDs, barriers and facilitators associated with guidelines' utilization were extracted from selected articles.

RESULTS: 8671 citations were retrieved, and 43 articles were selected. 51\% of studies were from Europe, and most were quantitative studies (64\%) following a cross-sectional design (88\%). Almost $80 \%$ of articles dealt with low back pain guidelines, and the most studied healthcare providers were general practitioners or physiotherapists. Five main barriers to guideline utilization were expressed by providers: 1) disagreement between recommendations and patient expectations; 2) guidelines not specific to individual patients; 3) unfamiliarity with "non-specific" term, or with the bio psychosocial model of MSDs; 4) time consuming; and 5) heterogeneity in guideline methods. Four main facilitators to guideline utilization were cited: 1) clinician's interest in evidence-based practice; 2) perception from clinicians that the guideline will improve triage, diagnosis and management; 3) time efficiency; and 4) standardized language.
\end{abstract}

Conclusion: Identifying modifiable determinants is the first step in developing implementation strategies to improve guideline utilization in clinical practice.

Keywords: Clinical practice guidelines - musculoskeletal disorders, Adherence

\footnotetext{
* Correspondence: d.sorondo@ifec.net

'UMR1295, Toulouse III University, Inserm, Equipe EQUITY, Equipe constitutive du CERPOP, Toulouse, France

${ }^{2}$ Institut Franco-Européen de Chiropraxie, 72 chemin de la Flambère-31,300,

Toulouse, France

Full list of author information is available at the end of the article
}

(c) The Author(s). 2021 Open Access This article is licensed under a Creative Commons Attribution 4.0 International License, which permits use, sharing, adaptation, distribution and reproduction in any medium or format, as long as you give appropriate credit to the original author(s) and the source, provide a link to the Creative Commons licence, and indicate if changes were made. The images or other third party material in this article are included in the article's Creative Commons licence, unless indicated otherwise in a credit line to the material. If material is not included in the article's Creative Commons licence and your intended use is not permitted by statutory regulation or exceeds the permitted use, you will need to obtain permission directly from the copyright holder. To view a copy of this licence, visit http://creativecommons.org/licenses/by/4.0/. The Creative Commons Public Domain Dedication waiver (http://creativecommons.org/publicdomain/zero/1.0/) applies to the data made available in this article, unless otherwise stated in a credit line to the data. 


\section{Contributions to the literature' statement}

- lack of studies focusing on both barriers and facilitators of all practitioners involved in the management of one pathology;

- No study has investigated the barriers and facilitators of guidelines' utilization in all healthcare practitioners managing MSDs;

- Here, the emphasis is putted on the management and the assessment of a disorder not depending on a profession;

- Providing an overview of determinants related to guideline utilization by health care providers;

- Odd situation: some determinants are both barriers and facilitators.

\section{Introduction}

Musculoskeletal disorders (MSDs) are the leading cause of years lived with disability in the world [1]. These disorders affect people of all ages but the prevalence peaks in older individuals [2]. The Centers for Disease Control and Prevention (CDC) define MSDs as grade I-II sprain/ strains, tendinitis, tendinosis, tendinopathy, neuropathies and nonspecific pain of the upper extremity, lower extremity, or spine [3]. MSDs can impact health-related quality of life, social interactions and work habilitations [2]. Consequently, MSDs have economic repercussions for patients and society [2].

Many clinical practice guidelines exist to inform the management of MSDs [4-9]. Guidelines are defined as guidance documents for clinical practice [10]. They are generally developed by synthesizing the best evidence on patient-centered care [5,6]. For that reason, clinicians are encouraged to use guidelines to improve: 1 ) health outcomes in patients, 2) quality of clinical decisions by healthcare professionals, 3) efficiency of the healthcare system, 4) safety of care, and 5) cost effectiveness [11].

Although encouraged, guideline utilization by clinicians is suboptimal [12], even if barriers and facilitators of guideline utilization and adherence have been identified in the literature [11-13]. In their framework Cabana et al. identified nine barriers involved in physicians' utilization: 1) lack of familiarity, 2) lack of awareness, 3) lack of agreement, 4) lack of self-efficacy, 5) lack of positive outcome expectancy, 6) lack of motivation, 7) external barriers, 8) patient-related barriers; and 9) contextrelated barriers. However, previous research focused on barriers related to one type of health care practitioners (mainly physicians) for one chronic disease such as diabetes, cancer, osteoarthritis, or low back pain [13].

Consequently, pathology and healthcare practitioners could be relevant factors (barriers or facilitators) of guidelines' utilization. There is a lack of studies focusing on both barriers and facilitators of all practitioners involved in the management of one pathology. To our knowledge, no study has investigated the barriers and facilitators of guidelines' utilization in all healthcare practitioners managing MSDs. This knowledge could inform implementation strategies. Therefore, we conducted a scoping review of the literature to describe the determinants of clinical practice guidelines' utilization for the assessment and management of MSDs.

\section{Method}

Our scoping review of the literature followed the methods proposed by Arksey and O'Malley [14-16] and included five steps: 1 ) identification of the research question; 2) identifying relevant studies; 3) selection of studies; 4) charting data with critical appraisal; and 5) collation, synthesis, and reporting results. Our review complies with the Reporting Items for Systematic Reviews and Meta-Analyzes extension for Scoping Reviews (PRISMA-ScR) statement [17]. We added one step to the methodology proposed by Arksey and O'Malley and critically appraised the methodological quality of relevant studies. Although quality assessment of studies is not yet a standard methodological step when conducting a scoping review, it is recommended in the PRISMA Scoping Review [17].

\section{Step 1: identifying the research question}

Our research question was: "What are the determinants of use of clinical practice guidelines by healthcare providers for the assessment and management of musculoskeletal disorders?"

\section{Step 2: identifying relevant studies}

A search strategy was developed in collaboration with a health-science librarian and reviewed by a second health-science librarian using the Peer Review of Electronic Search Strategies (PRESS) Checklist $[18,19]$ (See Appendix S1 for MEDLINE search strategy).

Three electronic databases (MEDLINE, Embase and AMED, through Ovid Technologies) were systematically searched from inception to March 12th, 2021. Search terms included subject headings specific to each database (e.g. MeSH in MEDLINE) and free text words relating to: "musculoskeletal disorders" AND "health practitioners" AND "guidelines". The search strategy was first developed in MEDLINE and then adapted to the other bibliographic databases. We used the PRISMA-ScR flow chart to report number of articles at each stage [17].

\section{Step 3: study selection Eligibility criteria}

Eligible articles met the following inclusion criteria: 1) peer-reviewed articles published in English, French or Spanish; 2) investigation of determinants of guideline 
utilization focusing on barriers and facilitators (any factors that influence the utilization of evidence-based musculoskeletal guidelines); 3) source population included healthcare providers involved in the management of musculoskeletal disorders; and 4) epidemiological (controlled trials, cohort or cross-sectional studies), qualitative or mixed-method study designs. We excluded: 1) cadaveric or animal studies; and 2) books, book reviews, book chapters, conference abstracts, conference papers, editorials or letters to the editor, and literature reviews.

\section{Screening}

Two reviewers (DS and NL) independently screened all articles in two phases. In phase 1 , reviewers screened titles and abstracts and classified articles as irrelevant, relevant, and possibly relevant. In phase 2 , the full text of potentially relevant articles was reviewed for eligibility. When reviewers disagreed, they discussed until reaching consensus. If consensus could not be reached, the article was independently screened by a third reviewer $(\mathrm{CD}$ or $\mathrm{PC})$ who discussed with the initial pair of reviewers to resolve disagreement.

\section{Step 4: charting data \\ Extraction of data}

The lead author (DS) extracted data from all relevant studies and built an evidence Table. A second reviewer (NL) checked the validity of the data extraction. Extracted data included: 1) article identification (first author name, publication date and country); 2) study design (cross-sectional, cohort, randomized controlled trial; qualitative or mixed methods); 3) type of healthcare provider (DC: Doctor of Chiropractic; DO: Doctor of Osteopathy; MD: Medical Doctor; OT: Occupational Therapist; PT: Physiotherapist); 4) type of musculoskeletal disorders (low back pain, whiplash, neck pain, upper limbs, lower limbs, mixed MSDs); 5) guidelines related barriers and facilitators; and 6) quality of the study (low, medium, high).

\section{Critical appraisal of studies}

Pairs of reviewers (DS and NL) independently critically appraised relevant studies. Internal validity consensus of articles among reviewers was reached through discussion. A third independent reviewer was involved when consensus could not be reached (CD). For qualitative studies, methodological quality was assessed using the COnsolidated criteria for REporting Qualitative research Checklist (COREQ) [20]. For quantitative studies, methodological quality was assessed using the critical appraisal tools developed by Salmi [21]. The tool allows the classification of various study designs according to their methodological quality in one of 4 levels of internal validity: very good, quite good, low but acceptable, and unacceptable.

According to this classification, if the article complied with all internal-validity criteria in the quality checklist, the level of internal validity was assessed as "very good". If the article missed some criteria but followed all major ones defined by the checklist, the level of internal validity was assessed as "quite good". If one or more of those major criteria were missing, the level of internal validity was noted as "low but acceptable". And, finally, if the article followed none of the criteria, the level of internal validity was "unacceptable".

We did not exclude articles based with unacceptable quality. Rather, we used internal validity as a criteria to stratify the synthesis and classified studies in 3 categories: 1) low internal validity (gathering low but acceptable and unacceptable levels of the checklist) a; 2) moderate internal validity (quite good internal validity); and 3 ) high internal validity (very good level).

\section{Step 5: collation, synthesis, and reporting the results} We extracted the following data and synthesized them according to the two following steps:

1) Study characteristics: first author, year of publication, country, study design, healthcare provider(s), musculoskeletal disorder(s), methodological quality.

Reported determinants of guidelines' use: barriers and facilitators were extracted and classified according to the theory of planned behavior (TPB) [22]. This theory is the most frequent used classification to understand how determinants could influence clinicians' practice [23]. This model approaches behavior by referring to three main concepts. The first concerns the attitudes and behavioral intention of healthcare providers toward guideline utilization. The second deals with the influence of subjective and social norms on guideline use. Finally, the third concept relates to perceived power and the perceived behavioral control.

Attitudes toward guideline use focusses on clinicians' cognitive and emotional beliefs about their behavior, and individual positive or negative evaluation of the use of guidelines. This part includes the intention to perform a given behavior and therefore refers to motivational factors that may influence a behavior: if you have a strong intention to perform the behavior, you will perform it.

The subjective norms refer to the perception of usual behavior by other peoples, and the perceived pressure to comply with this behavior, including an 
individual's motivation and cultural or social context influence.

The perceived behavioral control deals with the availability of skills needed to carry out the behavior, including expected external factors such as available resources and opportunities (e.g. skills, time, and cooperation of others). This part deals with factors linked with perceived ease or difficulty to perform a behavior.

These three concepts can influence each other. The more favorable the attitude and subjective norm with respect to a given behavior and the greater the perceived control, the stronger the intention to perform the behavior. Thus, barriers can be obstacles to the intention to perform the expected behavior - in our case guidelines utilization-and thus act as negative predictors of intention, because they contribute to an unfavorable evaluation of the use of guidelines. Obviously, facilitators act as positive predictors of intention and thus contribute to a favorable evaluation of the use of guidelines.

2) Interpretation of findings: We used mind mapping [24] to synthesize barriers or facilitators as a visual interpretation.

\section{Results}

Our search retrieved 8671 citations (Fig. 1). After removing duplicates $(n=1451)$, we screened 7220 articles. During the phase 1 screening, 7070 articles were irrelevant, and 150 full text were examined. During the phase 2 screening, 107 articles were excluded for the following reasons: 1) outcomes did not focus on guideline utilization $(n=86)$; 2) MSDs were not the only condition studied and the results were not stratified by disorders $(n=13)$; 3) publication type was not eligible (commentaries et poster presentation) $(n=7) ; 4)$ full-text article was not available $(n=1)$. Therefore, our synthesis included 43 articles (Table 1) [4, 25-60].

\section{Study characteristics}

Most articles $(67 \%, 29 / 43)$ were published after 2009, $30 \%(n=12)$ between 2001 and 2009, and 3\% $(n=2)$ were published before 2001 (Table 1). Studies were mainly conducted in Europe $(51 \%, n=22)$, and North America (35\%, $n=15)$, with a lower proportion elsewhere $(7 \%$ in New Zealand or Australia $(n=3), 5 \%$ in Israel $(\mathrm{n}=2)$ and $2 \%$ in Africa $(\mathrm{n}=1))$. Low back pain was the most studied disorder $(81 \%, n=35)$, followed by neck pain and associated disorders $(12 \%, n=5)$, lower limb disorders $(n=1)$, and two articles evaluated mixed MSDs. Most studies $(63 \%, n=27)$ used epidemiological designs, 30\% $(n=13)$ used qualitative designs and $7 \%$ $(\mathrm{n}=3)$ used mixed methods. Most epidemiological studies were cross-sectional $(85 \%, n=23), 11 \%(n=3)$ were cohorts, and one was a randomized controlled trial. Forty percent of studies $(n=17)$ investigated physiotherapists; 30\% ( $n=13)$ medical doctors; 9\% $(n=4)$ doctors of chiropractic; $2 \%(n=1)$ occupational therapists; $2 \%$ $(\mathrm{n}=1)$ doctors of osteopathy; and 16\% $(n=7)$ investigated multiple professions.

Regarding methodological quality, $42 \%(n=18), 30 \%$ $(n=12)$ and $30 \%(n=13)$ were of low, medium and highquality level, respectively.

Finally, we found no difference between determinants related to internal validity, country, type of healthcare providers or MSDs.

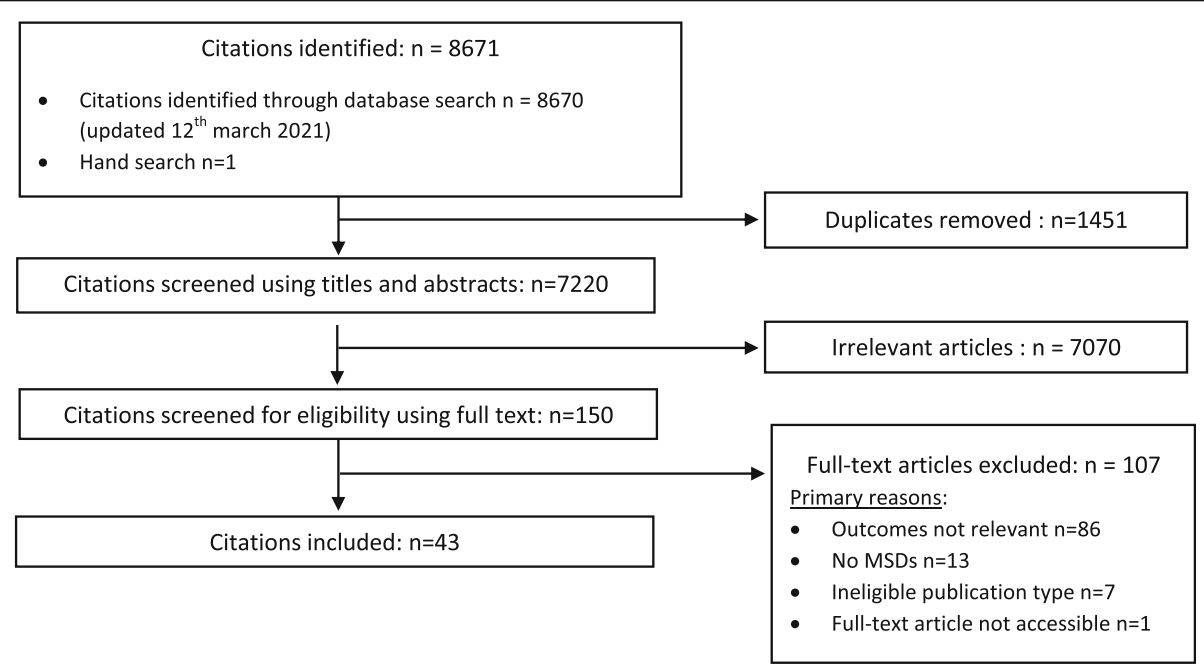

Fig. 1 Flow chart of articles screening 
Table 1 Description of articles included classified by year of publication ( $n=43$ )

\begin{tabular}{|c|c|c|c|c|c|}
\hline $\begin{array}{l}\text { First author } \\
\text { Year of publication }\end{array}$ & Country & Design & $\begin{array}{l}\text { Healthcare } \\
\text { providers }\end{array}$ & $\begin{array}{l}\text { Musculoskeletal } \\
\text { disorder (MSDs) }\end{array}$ & $\begin{array}{l}\text { Quality of } \\
\text { the study }\end{array}$ \\
\hline Biggs et al. 1997 [25] & North America & Quantitative (cross-sectional) & $D C(n=401)$ & Mixed MSDs & Low \\
\hline Schers et al. 2000 [26] & Europe & Quantitative (cohort) & $\mathrm{MD}(n=30)$ & Low back pain & High \\
\hline Hurley et al. 2002 [27] & North America & Quantitative (cross-sectional) & PT $(n=118)$ & Neck pain & Low \\
\hline Espeland et al. 2003 [28] & Europe & Qualitative & $\mathrm{MD}(n=13)$ & Low back pain & Low \\
\hline Schectman et al. 2003 [29] & North America & Quantitative (RCT) & $\mathrm{MD}(n=53)$ & Low back pain & Low \\
\hline Baker et al. 2006 [30] & Europe & Qualitative & $\mathrm{MD}(n=29)$ & Low back pain & Low \\
\hline Coudeyre et al. 2006 [31] & Europe & Quantitative (cross-sectional) & $\mathrm{MD}(n=864)$ & Low back pain & Low \\
\hline Dahan et al. 2006 [32] & Israel & Qualitative & $\mathrm{MD}(n=38)$ & Low back pain & Low \\
\hline Poiraudeau et al. 2006 [33] & Europe & Quantitative (cross-sectional) & $\mathrm{MD}(n=266)$ & Low back pain & Medium \\
\hline Bishop et al. 2008 [34] & Europe & Quantitative (cross-sectional) & $\begin{array}{l}\text { Mixed providers } \\
(n=1022)\end{array}$ & Low back pain & Medium \\
\hline Chenot et al. 2008 [35] & Europe & Quantitative (cross-sectional) & $\mathrm{MD}(n=72)$ & Low back pain & Low \\
\hline Corbett et al. 2009 [36] & Europe & Mixed method & $\mathrm{MD}(n=16)$ & Low back pain & Medium \\
\hline Côté et al. 2009 [37] & North America & Qualitative & $\mathrm{PT}(n=16)$ & Low back pain & High \\
\hline Harting et al. 2009 [38] & Europe & Qualitative & $\mathrm{PT}(n=30)$ & Low back pain & High \\
\hline Rutten et al. 2009 [39] & Europe & Quantitative (cross-sectional) & PT $(n=232)$ & Low back pain & Medium \\
\hline Fullen et al. 2011 [40] & Europe & Quantitative (cross-sectional) & $\mathrm{MD}(n=432)$ & Low back pain & High \\
\hline Kooijman et al. 2011 [41] & Europe & Quantitative (cross-sectional) & PT $(n=121)$ & Lower limb & Low \\
\hline Poitras et al. 2011 [42] & North America & Qualitative & OT $(n=9)$ & Low back pain & High \\
\hline Bussières et al. 2012 [4] & North America & Qualitative & $\mathrm{DC}(n=21)$ & Low back pain & High \\
\hline Jeffrey et al. 2012 [43] & North America & Qualitative & $\mathrm{PT}(n=11)$ & Low back pain & Low \\
\hline Pincus et al. 2012 [44] & Europe & Quantitative (cross-sectional) & $\begin{array}{l}\text { Mixed providers } \\
(n=465)\end{array}$ & Low back pain & Medium \\
\hline Poitras et al. 2012 [45] & North America & Qualitative & $\begin{array}{l}\text { Mixed providers } \\
(n=32)\end{array}$ & Low back pain & High \\
\hline Simmonds et al. 2012 [46] & North America & Quantitative (cross-sectional) & PT $(n=100)$ & Low back pain & Medium \\
\hline Matzon et al. 2013 [47] & Europe & Quantitative (cross-sectional) & PT $(n=187)$ & Low back pain & Low \\
\hline Hendrick et al. 2013 [48] & NZ-Australia & Quantitative (cross-sectional) & PT $(n=170)$ & Low back pain & Low \\
\hline Rebbeck et al. 2013 [49] & NZ-Australia & Quantitative (cohort) & $\begin{array}{l}\text { Mixed providers } \\
(n=80)\end{array}$ & Whiplash & High \\
\hline Corkery et al. 2014 [50] & North America & Quantitative (cross-sectional) & PT $(n=291)$ & Whiplash & Low \\
\hline Gremeaux et al. 2014 [51] & Europe & Quantitative (cross-sectional) & $\mathrm{MD}(n=47)$ & Low back pain & Medium \\
\hline Learman et al. 2014 [52] & North America & Quantitative (cross-sectional) & PT $(n=1144)$ & Low back pain & Medium \\
\hline Bishop et al. 2015 [53] & Europe & Qualitative & $\begin{array}{l}\text { Mixed providers } \\
(n=53)\end{array}$ & Low back pain & Medium \\
\hline Bernhardsson et al. 2015 [54] & Europe & Quantitative (cross-sectional) & PT $(n=271)$ & Mixed MSDs & Low \\
\hline Clement et al. 2015 [55] & North America & Quantitative (cross-sectional) & $\begin{array}{l}\text { Mixed providers } \\
(n=456)\end{array}$ & Neck pain & Low \\
\hline Maas et al. 2015 [56] & Europe & Mixed method & $\mathrm{PT}(n=44)$ & Low back pain & Low \\
\hline Ladeira et al. 2015 [57] & North America & Quantitative (cross-sectional) & PT $(n=327)$ & Low back pain & Medium \\
\hline Brijnath et al. 2016 [58] & North America & Quantitative (cross-sectional) & $\mathrm{MD}(n=423)$ & Whiplash & Medium \\
\hline Derghazarian et al. 2016 [59] & North America & Quantitative (cross-sectional) & PT $(n=108)$ & Low back pain & High \\
\hline Epstein-Sher et al. 2017 [60] & Israel & Quantitative (cross-sectional) & $\mathrm{MD}(n=86)$ & Low back pain & Low \\
\hline Figg-Latham et al. 2017 [61] & Europe & Qualitative & $\mathrm{DO}(n=12)$ & Low back pain & High \\
\hline Suman et al. 2017 [62] & Europe & Mixed method & $\begin{array}{l}\text { Mixed providers } \\
(n=96)\end{array}$ & Low back pain & Low \\
\hline
\end{tabular}


Table 1 Description of articles included classified by year of publication $(n=43)$ (Continued)

\begin{tabular}{|c|c|c|c|c|c|}
\hline $\begin{array}{l}\text { First author } \\
\text { Year of publication }\end{array}$ & Country & Design & $\begin{array}{l}\text { Healthcare } \\
\text { providers }\end{array}$ & $\begin{array}{l}\text { Musculoskeletal } \\
\text { disorder (MSDs) }\end{array}$ & $\begin{array}{l}\text { Quality of } \\
\text { the study }\end{array}$ \\
\hline Stilwell et al. 2017 [63] & North America & Qualitative & Dc $(n=13)$ & Low back pain & High \\
\hline Cowell et al. 2018 [64] & Europe & Qualitative & PT $(n=10)$ & Low back pain & Medium \\
\hline Selby et al. 2018 [65] & Europe & Quantitative (cross-sectional) & $\mathrm{GP}(n=167)$ & Low back pain & High \\
\hline Akindele et al. 2019 [66] & Africa & Quantitative (cross-sectional) & PT $(n=189)$ & Low back pain & High \\
\hline
\end{tabular}

DC Doctor of Chiropractic, DO Doctor of Osteopathy, MD Medical Doctor, MSDs Musculoskeletal Disorder, NZ New Zealand, OT Occupational Therapist, PT, Physiotherapist, $R C T$, Randomized Clinical Trial

\section{Barriers to guidelines' utilization}

Barriers to guidelines' utilization are reported in Fig. 2 according to their frequency of citation in the literature and detailed below according to the planned behavior theory.

\section{Barriers linked to clinicians' attitudes towards their behavior} Clinicians who feel frustrated, anxious or a perceived loss of autonomy when using guidelines were less likely to use them [4, 27, 28, 32, 38, 43, 63, 64, 67]. Furthermore, being afraid of missing information such as clinical signs or patient information, was also perceived as a barrier to use guidelines $[4,26,28,32]$. The perception of a gap between the biopsychosocial model of care recommended by guidelines and their current practice (for example biomedical approach) $[28,31,33,51,63,64]$, a culture of suspicion about guidelines [36], and a skeptical view of medicine or evidence-based practice [53,61] were identified as barriers of guideline utilization.

\section{Barriers included in subjective norms}

We identified barriers related to clinicians' judgment and perception of their own behavior by other people. Health care providers reported to be influenced by noncompliance of their instructors during their training [4, 61], non-compliance of colleagues or other professionals in their practice [4, 28, 30, 32, 36, 45, 55, 67]. For example, when they had experienced the feeling of being in competition with other practitioners $[36,61]$. Moreover, authorities and public health policies could have an impact on clinicians perceiving guidelines as mandatory [36]. Finally, long-term patients could also have an impact on guidelines' utilization; clinicians could be afraid to lose these patients if they do not satisfy their expectations [30, 35, 61, 63-65].

Barriers involved in clinicians perceived behavioral control Barriers were related to clinicians' perception of how they control their behavior. Guidelines may be perceived as

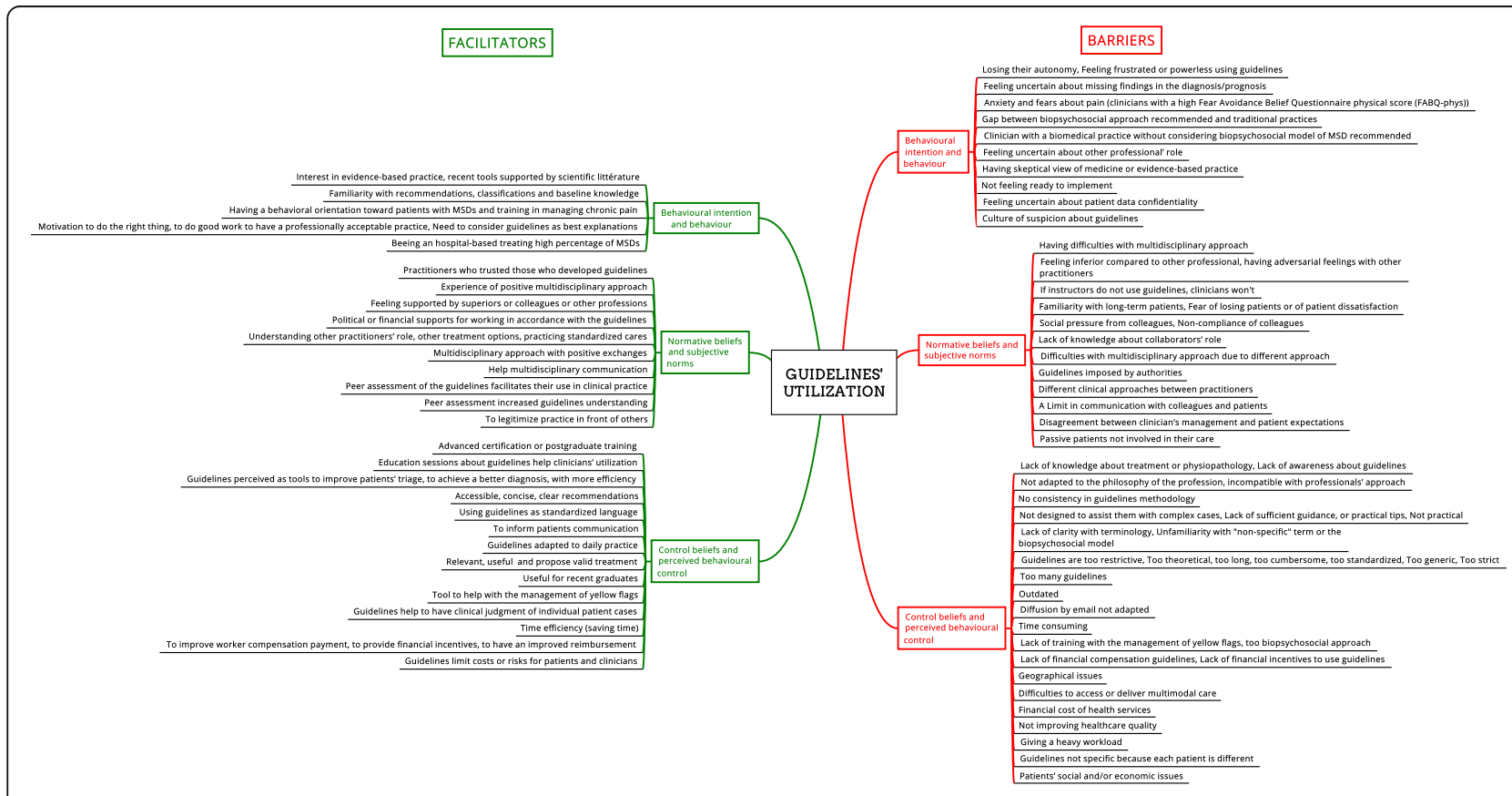

Fig. 2 Determinants of guidelines 'utilization emerging from the literature 
non-practical for current practice $[26,28,29,32,41,45$, $58,62,64-66]$ or reported to be too restrictive, theoretical, long, cumbersome $[4,27,37,38,53,56,63,64,66,67]$ or outdated $[61,67]$. The number of available guidelines may be viewed as too large for practitioners [36, 38], with a lack of consistency in their methodology $[4,28,38,42,45$, 66, 67]. Consequently, clinicians may be confused when selecting a guideline. Furthermore, terminology used is sometimes perceived to be unclear, particularly regarding the term "non-specific" used to describe some MSDs such as neck or low back pain [32, 37, 38, 43, 45, 53, 60, 62].

In addition, some clinicians reported that they are not sufficiently trained to use guideline recommendations. For example, clinicians who are not trained to use yellow flags, or the biopsychosocial approach would be challenged with using them to manage patients $[30,34,37$, $42,45,46,48,59,63,64,67]$. Barriers to compliance also include the ability to provide recommended multimodal care, and accessibility and reimbursement for healthcare services [30, 32, 35, 42, 45, 53, 58, 66, 67]. For these reasons, some practitioners perceived guidelines as not adapted to the needs of their patients, limiting the interest in using guidelines to inform care for individual cases.

\section{Facilitators to guidelines' utilization}

All facilitators to guideline utilization are reported in Fig. 2.

\section{Facilitators linked to clinicians' attitudes towards their behavior} A practitioner's motivation to provide good clinical care, positive behaviors toward using guidelines and professionalism were frequently associated with compliance $[4$, $28,35]$. Providers interested in scientific literature used guidelines more frequently [4, 32, 36, 42, 50, 53, 67]. Clinicians who are interested in evidence-based practice are more likely to use recommendations from guidelines $[49,50,60]$. Furthermore, clinicians practicing in a hospital or a clinic with a large volume of MSDs patients reported using guidelines more often $[41,52,57]$.

\section{Facilitators included in subjective norms}

These determinants involved how clinicians practice and interact with others [62]. Relationships and social interactions with colleagues, superiors, and public health authorities can influence guideline utilization $[39,45,55$, $56,63,66]$. Moreover, clinicians who use recommendations prefer to perceive that the guidelines are commonly used in practice by others in their field [37, 42]. Having good experiences with recommended multidisciplinary approaches [62] encourages clinicians to maintain this behavior in practice. In this way, they use guidelines as a common and shared language between different professions $[38,39,42,61]$. Clinicians who want to legitimize their own practice in front of others [61] use guidelines in practice. Finally, it is reported that clinicians need to trust those who developed guidelines $[4,38]$ and must have financial resources supported by authorities to work in accordance with guidelines [38, $39,63]$.

\section{Facilitators involved in clinicians' perceived behavioral control}

Determinants may be linked to clinicians' perception of control about their ability to use guidelines. Recommendations must be perceived as accessible, concise, clear, adapted to daily practice, useful and relevant for use by clinicians [32, 37, 39, 42, 63, 67].

Providers who aim to improve their practice tend to use more guidelines. Practice improvement occurs when providers view guidelines $\mathrm{s}$ tools to help form clinical judgments, inform patient communication, improve patients' triage, and be more efficient $[35,39,45,53,55$, $58,63]$. Some clinicians expressed the need to be trained by having access to education sessions about guidelines utilization $[29,66]$.

\section{Discussion}

\section{Summary of evidence}

Our scoping review provides an overview of determinants related to guideline utilization by health care providers. Most studies focusing on guideline utilization used a cross-sectional design. Barriers to guideline utilization were more common than facilitators. We did not find any difference between determinants influencing utilization of guidelines related to healthcare provider or MSDs. This suggests that barriers and facilitators highlighted in this scoping review may apply to various healthcare providers who manage MSDs. The majority of determinants identified in this review were reported from high or moderate internal validity studies.

We note that only a few determinants associated with patient characteristics have been identified, underscoring the fact that the literature on guideline use is largely focused on clinician characteristics.

Our results suggest that obstacles and facilitators tend to hold opposite views. For example, guideline users perceive them as adaptable to daily practice because they are relevant, useful, accessible, concise and clear. Alternatively, non-users perceive guidelines as not improving healthcare quality because they are restrictive, cumbersome, theoretical, too plentiful, and time consuming.

According to clinicians' behavior, two concepts that influence guideline utilization depend on a clinician's perception of the factor (Fig. 2). The first concept is a positive practice orientation such as feeling comfortable with the management of yellow flags and biopsychosocial factors which are supported by guidelines. On the 
other hand, clinicians with a biomedical practice seem to have difficulties considering the biopsychosocial model recommended by guidelines. Second, the habit of working with this kind of tool is important. We can differentiate between clinicians who are familiar with recommendations or classifications and those who are not, and consequently feel frustrated or powerless using these tools.

We found that perception of clinical behavior by colleagues, other professionals or authorities could have both negative and positive influence on clinicians' guideline utilization. Moreover, past experiences can enhance or limit the use of guidelines. Having a positive experience with others when guidelines recommend multidisciplinary approaches in the management of a patient can encourage practitioners to repeat the experience and apply guidelines. On the contrary, when clinicians feel at a disadvantage compared to other professions, or experience disagreement without explanations about multidisciplinary management of a patient, they may not use guidelines. Finally, authorities impact the use of guidelines through the diffusion of tools and by adding value to clinical management following guideline recommendations. Clinicians use guidelines if they feel supported to work in accordance with recommendations. If they feel they are not supported and perceive guidelines as imposed, their attitude toward the use of guidelines is negative.

\section{Comparison with previous reviews of the literature}

Our results concerning barriers to guideline utilization agree with previous studies $[13,68]$. Cabana et al. also reported that barriers to guideline use include: lack of awareness, lack of familiarity, lack of agreement with specific guidelines or guidelines in general, lack of selfefficacy, lack of outcome expectancy, lack of motivation, external barriers linked with patient factors, guideline factors or environmental factors [13]. Furthermore, in their scoping review, Fischer et al. identified the following barriers among physicians: lack of awareness, a lack of familiarity, lack of agreement, self-efficacy, skills, outcome expectancy and motivation [68]. However, our scoping review adds to the literature by identifying a complete list of barriers and facilitators of guideline utilization in a broad range of health care providers who manage patients with MSDs. Importantly, our review found no association between determinants and professions. Our determinants are classified using a standardized framework and visually displayed in a mind-map (Fig. 2).

The evidence included in our scoping review suggest that the recommendations focus on altering the behaviors of guidelines consumers. However, one possible barrier which was not discussed in the literature is that the guidelines themselves are often inconsistent in their methodology and recommendations. Therefore, we recommend that the AGREE reporting checklist and RIGH $T$ statement be used for the reporting of clinical practice guidelines [69, 70].

\section{Strengths and limitations}

A strength of our scoping review is its comprehensive scope. We focused our research on all MSDs to emphasize the importance of patient-centered care for these disorders in a multidisciplinary approach. This clinical situation involves all healthcare providers and data were described from a clinical point of view.

We used the Theory of Planned Behavior. This is the most frequently classification used in implementation science [13]. This theory helps identify healthcare providers' determinants of intension to use, their use of guidelines, and their beliefs towards guidelines. This is a major step in developing targeted interventions for clinicians' adherence. Future interventions must focus on maintaining, sustaining and encouraging guideline use, and thus encourage positive behaviors that improve diagnosis and patient management. Finally, we performed a critical appraisal, a limitation mentioned in previous scoping reviews on this topic [17]. Our critical appraisal revealed that two thirds of determinants were extracted from high or medium quality studies. One third of determinants, therefore, needs to be carefully considered. Our scoping review has some limitations. Because we searched only three databases (adhering to standard scoping review methodology), we may have missed some studies. Also, we did not search the grey literature, and we restricted languages to English, Spanish or French.

\section{Conclusion and perspectives}

Implementation strategies need to be oriented toward determinants expressed by clinicians, the major users of guidelines. Implementation tools must be developed that are tailored to clinicians' expectations and that are informed by facilitators of utilization and that avoid barriers to orient work on implementation strategies. Our study identifies determinants for public health policy makers and professional associations that need to be prioritized when implementing guidelines. Whether the use of guidelines varies according to the social characteristics of patients could also be an interesting question for further research.

\section{Supplementary Information}

The online version contains supplementary material available at https://doi. org/10.1186/s12891-021-04204-w.

Additional file 1: Appendix 1. Search strategy in Medline. 


\section{Acknowledgements}

The authors acknowledge and thank Mr. Hainan Yu and Dr. Margareta Nordin, for their help and suggestions.

\section{Authors' contributions}

DS, CD, PC, and NL developed the research questions and scope of the study. DS and NL conducted phase 1 and 2 screening and data charting. DS drafted the manuscript, prepare tables and figure with NL's contribution. ATV developed the literature search strategies in collaboration with the other authors. DS, NL, CD, PC, LRS, and CC contributed to the organization, analysis, and interpretation of the results. All authors read and approved the final manuscript.

\section{Funding}

This study was funded by the Institut Franco-Européen de Chiropraxie in a $\mathrm{PhD}$ project. This association was not involved in the collection of data, data analysis, interpretation of data, or drafting of the manuscript.

\section{Availability of data and materials}

Raw data are available on request to the first author dsorondo@ifec.net

\section{Declarations}

\section{Ethics approval and consent to participate}

Not applicable.

\section{Consent for publication}

Not applicable.

\section{Competing interests}

The authors declare that they have no conflict of interest.

\section{Author details}

'UMR1295, Toulouse III University, Inserm, Equipe EQUITY, Equipe constitutive du CERPOP, Toulouse, France. ${ }^{2}$ Institut Franco-Européen de Chiropraxie, 72 chemin de la Flambère-31,300, Toulouse, France. ${ }^{3}$ Faculty of Health Sciences, Ontario Tech University, Oshawa, Ontario, Canada. ${ }^{4}$ Centre for Disability Prevention and Rehabilitation at Ontario Tech University and the Canadian Memorial Chiropractic College, Oshawa and Toronto, Ontario, Canada. ${ }^{5}$ Univ. Bordeaux, ISPED, Centre INSERM U1219-Bordeaux Population Health, F-33000 Bordeaux, France. ${ }^{6} \mathrm{CHU}$ de Bordeaux, Pôle de santé publique, Service d'Information Médicale, F-33000 Bordeaux, France. ${ }^{7}$ Division of General Medical Rehabilitation, University of Geneva, Geneva, Switzerland. ${ }^{8}$ Division of Clinical Pharmacology and Toxicology, Multidisciplinary Pain Centre, Geneva University Hospitals, Geneva, Switzerland.

Received: 1 December 2020 Accepted: 25 March 2021

Published online: 01 June 2021

\section{References}

1. Green BN, Johnson CD, Haldeman S, Griffith E, Clay MB, Kane EJ, et al. A scoping review of biopsychosocial risk factors and co-morbidities for common spinal disorders. PLoS One. 2018;13(6):e0197987 https://doi.org/1 0.1371/journal.pone.0197987.

2. Global, regional, and national incidence, prevalence, and years lived with disability for 328 diseases and injuries for 195 countries, 1990-2016. A systematic analysis for the Global Burden of Disease Study 2016. Lancet. 2017;390(10100):1211-59.

3. CDC - Musculoskeletal disorders definition - https://www.cdc.gov/workpla cehealthpromotion/health-strategies/musculoskeletal-disorders/ (consulted: February 15th, 2021).

4. Bussieres AE, Patey AM, Francis JJ, Sales AE, Grimshaw JM, Brouwers M, et al. Identifying factors likely to influence compliance with diagnostic imaging guideline recommendations for spine disorders among chiropractors in North America: a focus group study using the theoretical domains framework. Implement Sci. 2012;7(1):82 https:/doi.org/10.1 186/1748-5908-7-82.

5. Wong JJ, Côté P, Sutton DA, Randhawa K, Yu H, Varatharajan S, et al. Clinical practice guidelines for the noninvasive management of low back pain: a systematic review by the Ontario protocol for traffic injury management (OPTIMa) collaboration. Eur J Pain. 2017;21(2):201-16 https://doi.org/10.1 002/ejp.931.
6. Côté P, Wong JJ, Sutton D, Shearer HM, Mior S, Randhawa K, et al. Management of neck pain and associated disorders: a clinical practice guideline from the Ontario protocol for traffic injury management (OPTIMa) collaboration. Eur Spine J. 2016;25(7):2000-22 https://doi.org/10.1007/ s00586-016-4467-7.

7. Chou R, Qaseem A, Snow V, Casey D, Cross JT Jr, Shekelle P, et al. Diagnosis and treatment of low back pain: a joint clinical practice guideline from the American College of Physicians and the American pain society. Ann Intern Med. 2007;147(7):478-91 https://doi.org/10.7326/0003-4819-147-7-20071002 0-00006.

8. Nielens H, Van Zundert J, Mairiaux P, Gailly J, Van Den Hecke N, Mazina D, et al. Chronic low back pain. Good Clinical practice (GCP). Brussels: Belgian Health Care Knowledge Centre (KCE); 2006. KCE reports 48 C (D/2006/10. 273/71).

9. de Campos TF. Low back pain and sciatica in over 16s: assessment and management NICE guideline [NG59]. J Physiother. 2017;63(2):120 https://doi. org/10.1016/j.jphys.2017.02.012

10. National Center for Complementary and Integrative Health - https://www. nccih.nih.gov/health/providers/clinicalpractice - (consulted: February 15th, 2021).

11. Woolf SH, Grol R, Hutchinson A, Eccles M, Grimshaw J. Clinical guidelines: potential benefits, limitations, and harms of clinical guidelines. BMJ. 1999; 318(7182):527-30 https://doi.org/10.1136/bmj.318.7182.527.

12. Samaan Z, Mbuagbaw L, Kosa D, Borg Debono V, Dillenburg R, Zhang S, et al. A systematic scoping review of adherence to reporting guidelines in health care literature. J Multidiscip Healthc. 2013;6:169-88 https://doi.org/1 0.2147/JMDH.S43952.

13. Cabana MD, Rand CS, Powe NR, Wu AW, Wilson MH, Abboud PA, et al. Why don't physicians follow clinical practice guidelines? A framework for improvement. JAMA. 1999;282(15):1458-65 https://doi.org/10.1001/jama.2 82.15.1458.

14. Arksey H, O'Malley L. Scoping studies: towards a methodological framework Int J Soc Res Methodol. 2005;8(1):19-32 https://doi.org/10.1080/1364557032 000119616.

15. Levac D, Colquhoun H, O'Brien KK. Scoping studies: advancing the methodology. Implement Sci. 2010;5(1):69 https://doi.org/10.1186/17485908-5-69.

16. O'Brien KK, Colquhoun H, Levac D, Baxter L, Tricco AC, Straus S, et al. Advancing scoping study methodology: a web-based survey and consultation of perceptions on terminology, definition and methodological steps. BMC Health Serv Res. 2016;16(1):305 https://doi.org/10.1186/s12913-01 6-1579-z.

17. Tricco AC, Lillie E, Zarin W, O'Brien KK, Colquhoun H, Levac D, et al. PRISMA extension for scoping reviews (PRISMA-SCR): checklist and explanation. Ann Intern Med. 2018;169(7):467-73 https://doi.org/10.7326/M18-0850.

18. McGowan J, Sampson M, Salzwedel DM, Cogo E, Foerster V, Lefebvre C. PRESS peer review of electronic search strategies: 2015 guideline statement. J Clin Epidemiol. 2016;75:40-6 https://doi.org/10.1016/j.jclinepi.2016.01.021.

19. Sampson M, McGowan J, Cogo E, Grimshaw J, Moher D, Lefebvre C. An evidence-based practice guideline for the peer review of electronic search strategies. J Clin Epidemiol. 2009;62(9):944-52 https://doi.org/10.1016/j. jclinepi.2008.10.012.

20. Tong A, Sainsbury P, Craig J. Consolidated criteria for reporting qualitative research (COREQ): a 32-item checklist for interviews and focus groups. Int J Qual Health Care. 2008;19:349-57.

21. Salmi RL. Lecture critique et communication médicale scientifique. Comment lire, présenter, rédiger et publier une étude clinique ou épidémiologique. 3e édition ed. Issy-les-Moulineaux. France: Elsevier Masson SAS; 2012.

22. Ajzen I. The theory of planned behavior. Organ Behav Hum Decis Process. 1991;50(2):179-211 https://doi.org/10.1016/0749-5978(91)90020-T.

23. Liang L, Abi Safi J, Gagliardi AR. Number and type of guideline implementation tools varies by guideline, clinical condition, country of origin, and type of developer organization: content analysis of guidelines. Implement Sci. 2017;12(1):136 https://doi.org/10.1186/s13012-017-0668-7.

24. XMind. (n.d.). Version XMind 8 Update 8 - Retrieved from http://www xmind.net.

25. Biggs L, Hay D, Mierau D. Canadian chiropractors' attitudes towards chiropractic philosophy and scope of practice: implications for the implementation of clinical practice guidelines. J Can Chiropr Assoc. 1997; 41(3):145-54. 
26. Schers H, Braspenning J, Drijver R, Wensing M, Grol R. Low back pain in general practice: reported management and reasons for not adhering to the guidelines in the Netherlands. Br J Gen Pract. 2000;50(457):640-4.

27. Hurley L, Yardley K, Gross AR, Hendry L, McLaughlin L. A survey to examine attitudes and patterns of practice of physiotherapists who perform cervical spine manipulation. Man Ther. 2002;7(1):10-8 https://doi.org/10.1054/math.2 001.0430 .

28. Espeland A, Baerheim A. Factors affecting general practitioners' decisions about plain radiography for back pain: implications for classification of guideline barriers--a qualitative study. BMC Health Serv Res. 2003;3(1):8 https://doi.org/10.1186/1472-6963-3-8.

29. Schectman JM, Schroth WS, Verme D, Voss JD. Randomized controlled trial of education and feedback for implementation of guidelines for acute low back pain. J Gen Intern Med. 2003;18(10):773-80 https://doi.org/10.1046/j.1 525-1497.2003.10205.x

30. Baker R, Lecouturier J, Bond S. Explaining variation in GP referral rates for $x$ rays for back pain. Implement Sci. 2006;1(1):15 https://doi.org/10.1186/17485908-1-15.

31. Coudeyre E, Rannou F, Tubach F, Baron G, Coriat F, Brin S, et al. General practitioners' fear-avoidance beliefs influence their management of patients with low back pain. Pain. 2006;124(3):330-7 https://doi.org/10.1016/j.pain.2 006.05.003.

32. Dahan R, Borkan J, Brown JB, Reis S, Hermoni D, Harris S. The challenge of using the low back pain guidelines: a qualitative research. J Eval Clin Pract. 2007;13(4):616-20 https://doi.org/10.1111/j.1365-2753.2007.00855.x

33. Poiraudeau S, Rannou F, Le Henanff A, Coudeyre E, Rozenberg S, Huas D, et al. Outcome of subacute low back pain: influence of patients' and rheumatologists' characteristics. Rheumatology (Oxford). 2006;45(6):718-23 https://doi.org/10.1093/rheumatology/kei231.

34. Bishop A, Foster NE, Thomas E, Hay EM. How does the self-reported clinical management of patients with low back pain relate to the attitudes and beliefs of health care practitioners? A survey of UK general practitioners and physiotherapists. Pain. 2008;135(1-2):187-95 https://doi.org/10.1016/j.pain.2 007.11.010.

35. Chenot JF, Scherer M, Becker A, Donner-Banzhoff N, Baum E, Leonhardt C, et al. Acceptance and perceived barriers of implementing a guideline for managing low back in general practice. Implement Sci. 2008;3(1):7 https:// doi.org/10.1186/1748-5908-3-7.

36. Corbett M, Foster N, Ong BN. GP attitudes and self-reported behaviour in primary care consultations for low back pain. Fam Pract. 2009;26(5):359-64 https://doi.org/10.1093/fampra/cmp042.

37. Côté AM, Durand MJ, Tousignant M, Poitras S. Physiotherapists and use of low back pain guidelines: a qualitative study of the barriers and facilitators. J Occup Rehabil. 2009;19(1):94-105 https://doi.org/10.1007/s10926-009-9167-2.

38. Harting J, Rutten GM, Rutten ST, Kremers SP. A qualitative application of the diffusion of innovations theory to examine determinants of guideline adherence among physical therapists. Phys Ther. 2009;89(3):221-32 https:// doi.org/10.2522/ptj.20080185.

39. Rutten G, Kremers S, Rutten S, Harting J. A theory-based cross-sectional survey demonstrated the important role of awareness in guideline implementation. J Clin Epidemiol. 2009;62(2):167-76 e1 https://doi.org/10.1 016/j.jclinepi.2008.04.004.

40. Fullen BM, Baxter GD, Doody C, Daly LE, Hurley DA. General practitioners' attitudes and beliefs regarding the management of chronic low back pain in Ireland: a cross-sectional national survey. Clin J Pain. 2011;27(6):542-9 https://doi.org/10.1097/AJP.0b013e31821771e2.

41. Kooijman MK, Swinkels IC, Veenhof C, Spreeuwenberg P, Leemrijse CJ. Physiotherapists' compliance with ankle injury guidelines is different for patients with acute injuries and patients with functional instability: an observational study. J Physiother. 2011;57(1):41-6 https://doi.org/10.1016/S1 836-9553(11)70006-6.

42. Poitras S, Durand MJ, Côté AM, Tousignant M. Use of low-back pain guidelines by occupational therapists: a qualitative study of barriers and facilitators. Work. 2011;39(4):465-75 https://doi.org/10.3233/WOR-2011-1196.

43. Jeffrey JE, Foster NE. A qualitative investigation of physical therapists' experiences and feelings of managing patients with nonspecific low back pain. Phys Ther. 2012;92(2):266-78 https://doi.org/10.2522/ptj.20100416.

44. Pincus T, Foster NE, Vogel S, Santos R, Breen A, Underwood M. Attitudes to back pain amongst musculoskeletal practitioners: a comparison of professional groups and practice settings using the ABS-mp. Man Ther. 2007;12(2):167-75 https://doi.org/10.1016/j.math.2006.06.005.
45. Poitras S, Durand MJ, Côté AM, Tousignant M. Guidelines on low back pain disability: interprofessional comparison of use between general practitioners, occupational therapists, and physiotherapists. Spine. 2012; 37(14):1252-9 https://doi.org/10.1097/BRS.0b013e31824b6adf.

46. Simmonds MJ, Derghazarian T, Vlaeyen JW. Physiotherapists' knowledge, attitudes, and intolerance of uncertainty influence decision making in low back pain. Clin J Pain. 2012;28(6):467-74 https://doi.org/10.1097/AJP.0b013 e31825bfe65.

47. Matzon JL, Lutsky KF, Maloney M, Beredjiklian PK. Adherence to the AAOS upper-extremity clinical practice guidelines. Orthopedics. 2013;36(11):e140711 https://doi.org/10.3928/01477447-20131021-22.

48. Hendrick P, Mani R, Bishop A, Milosavljevic S, Schneiders AG. Therapist knowledge, adherence and use of low back pain guidelines to inform clinical decisions--a national survey of manipulative and sports physiotherapists in New Zealand. Man Ther. 2013;18(2):136-42 https://doi. org/10.1016/j.math.2012.09.002.

49. Rebbeck T, Macedo LG, Maher CG. Compliance with clinical guidelines for whiplash improved with a targeted implementation strategy: a prospective cohort study. BMC Health Serv Res. 2013;13(1):213 https://doi.org/10.1186/14 72-6963-13-213

50. Corkery MB, Edgar KL, Smith CE. A survey of physical therapists' clinical practice patterns and adherence to clinical guidelines in the management of patients with whiplash associated disorders (WAD). J Man Manip Ther. 2014;22(2):75-89 https://doi.org/10.1179/2042618613Y.0000000048.

51. Gremeaux V, Coudeyre E, Viviez T, Bousquet PJ, Dupeyron A. Do teaching general Practitioners' fear-avoidance beliefs influence their Management of Patients with low Back pain? Pain Pract. 2015;15(8):730-7 https://doi.org/1 0.1111/papr.12248.

52. Learman KE, Ellis AR, Goode AP, Showalter C, Cook CE. Physical therapists' clinical knowledge of multidisciplinary low back pain treatment guidelines. Phys Ther. 2014;94(7):934-46 https://doi.org/10.2522/ptj.20130567.

53. Bishop FL, Dima AL, Ngui J, Little P, Moss-Morris R, Foster NE, et al. "lovely pie in the sky plans": a qualitative study of Clinicians' perspectives on guidelines for managing low Back pain in primary Care in England. Spine. 2015;40(23):1842-50 https://doi.org/10.1097/BRS.0000000000001215.

54. Bernhardsson S, Oberg B, Johansson K, Nilsen P, Larsson ME. Clinical practice in line with evidence? A survey among primary care physiotherapists in western Sweden. J Eval Clin Pract. 2015;21(6):1169-77 https://doi.org/10.1111/jep.12380.

55. Clement CM, Stiell IG, Lowe MA, Brehaut JC, Calder LA, Vaillancourt C, et al. Facilitators and barriers to application of the Canadian C-spine rule by emergency department triage nurses. Int Emerg Nurs. 2016;27:24-30 https://doi.org/10.1016/j.ienj.2015.11.008.

56. Maas MJ, van Dulmen SA, Sagasser MH, Heerkens YF, van der Vleuten CP Nijhuis-van der Sanden MW, et al. Critical features of peer assessment of clinical performance to enhance adherence to a low back pain guideline for physical therapists: a mixed methods design. BMC Med Educ. 2015; 15:203.

57. Ladeira CE, Samuel Cheng M, Hill CJ. Physical therapists' treatment choices for non-specific low back pain in Florida: an electronic survey. J Man Manip Ther. 2015;23(2):109-18 https://doi.org/10.1179/2042618613Y. 0000000065.

58. Brijnath B, Bunzli S, Xia T, Singh N, Schattner P, Collie A, et al. General practitioners knowledge and management of whiplash associated disorders and post-traumatic stress disorder: implications for patient care. BMC Fam Pract. 2016;17(1):82 https://doi.org/10.1186/s12875-016-0491-2.

59. Derghazarian T, Simmonds MJ. Management of low back pain by physical therapists in Quebec: how are we doing? Physiother Can. 2011;63(4):464-73 https://doi.org/10.3138/ptc.2010-04P.

60. Epstein-Sher S, Jaffe DH, Lahad A. Are they complying? Physicians' knowledge, attitudes, and readiness to change regarding low Back pain treatment guideline adherence. Spine. 2017:42(4):247-52 https://doi.org/10.1 097/BRS.0000000000001714.

61. Figg-Latham J, Rajendran D. Quiet dissent: the attitudes, beliefs and behaviours of UK osteopaths who reject low back pain guidance - a qualitative study. Musculoskelet Sci Pract. 2017;27:97-105 https://doi.org/1 0.1016/j.math.2016.10.006.

62. Suman A, Schaafsma FG, Buchbinder R, van Tulder MW, Anema JR. Implementation of a multidisciplinary guideline for low Back pain: processevaluation among health care professionals. J Occup Rehabil. 2017;27(3): 422-33 https://doi.org/10.1007/s10926-016-9673-y. 
63. Stilwell P, Hayden J, Rosiers P, Harman K, French S, Curran J, et al. A qualitative study of doctors of chiropractic in a Nova Scotian practice-based research network: barriers and facilitators to the screening and management of psychosocial factors for patients with low back pain. J Manip Physiol Ther. 2018;41(1):25-33 https://doi.org/10.1016/j.jmpt.2017.07. 014.

64. Cowell F, Gillespie S, Cheung G, Brown D. Complex regional pain syndrome in distal radius fractures: how to implement changes to reduce incidence and facilitate early management. J Hand Ther. 2018;31(2):201-5 https://doi. org/10.1016/j.jht.2018.01.013.

65. Selby K, Cornuz J, Cohidon C, Gaspoz JM, Senn N. How do Swiss general practitioners agree with and report adhering to a top-five list of unnecessary tests and treatments? Results of a cross-sectional survey. Eur Gen Pract. 2018;24(1):32-8 https://doi.org/10.1080/13814788.2017.1395018.

66. Akindele M, Rabiu M, Useh E. Assessment of the awareness, adherence, and barriers to low back pain clinical practice guidelines by practicing physiotherapists in a low-resourced country. Physiother Res Int. 2020;25(1): e1811 https://doi.org/10.1002/pri.1811. Epub 2019 Oct 21.

67. Parr S, May S. Do musculoskeletal physiotherapists believe the NICE guidelines for the management of non-specific LBP are practical and relevant to their practice? A cross sectional survey. Physiotherapy. 2014; 100(3):235-41 https://doi.org/10.1016/.jphysio.2013.09.004.

68. Fischer F, Lange K, Klose K, Greiner W, Kraemer A. Barriers and Strategies in Guideline Implementation-A Scoping Review. Healthcare (Basel). 2016;4(3): 36 Published 2016 Jun 29. https://doi.org/10.3390/healthcare4030036.

69. Brouwers MC, Kerkvliet K, Spithoff K. The AGREE reporting checklist: a tool to improve reporting of clinical practice guidelines. BMJ. 2016;352:11 152.

70. Chen Y, Yang K, Marušic A, Qaseem A, Meerpohl JJ, Flottorp S, et al. A reporting tool for practice guidelines in health care: the RIGHT statement. Ann Intern Med. 2017;166(2):128-32 https://doi.org/10.7326/M16-1565.

\section{Publisher's Note}

Springer Nature remains neutral with regard to jurisdictional claims in published maps and institutional affiliations.

Ready to submit your research? Choose BMC and benefit from:

- fast, convenient online submission

- thorough peer review by experienced researchers in your field

- rapid publication on acceptance

- support for research data, including large and complex data types

- gold Open Access which fosters wider collaboration and increased citations

- maximum visibility for your research: over $100 \mathrm{M}$ website views per year

At $\mathrm{BMC}$, research is always in progress.

Learn more biomedcentral.com/submissions 